\title{
Free Radical Shadow Cure Initiated Using Two-Component and Three-Component Initiator Systems
}

\author{
Hajime Kitano, ${ }^{1,2}$ Karthik Ramachandran, ${ }^{2}$ and Alec B. Scranton ${ }^{2}$ \\ ${ }^{1}$ Bridgestone Corporation, 3-1-1 Ogawahigashi-cho, Kodaira-shi, Tokyo 187-8531, Japan \\ ${ }^{2}$ Department of Chemical and Biochemical Engineering, The University of Iowa, Iowa City, IA 52242-1219, USA
}

Correspondence should be addressed to Alec B. Scranton, alec-scranton@uiowa.edu

Received 29 November 2011; Accepted 3 February 2012

Academic Editor: L. Maria Gómez

Copyright (C 2012 Hajime Kitano et al. This is an open access article distributed under the Creative Commons Attribution License, which permits unrestricted use, distribution, and reproduction in any medium, provided the original work is properly cited.

\begin{abstract}
In photopolymerization systems, "shadow cure" may be defined as polymerization which extends into regions which are not illuminated by the incident initiating light source. The objective of this study is to evaluate the use of fluorescent additives for polymerization in masked regions that are unilluminated by the incident initiating light. Two different fluorescent dyes are investigated: fluorescein (FL) and eosin Y spirit soluble (EYss). A systematic series of studies was performed to characterize the effects of fluorescence intensity, the incident light intensity, and the presence of a diphenyl iodonium salt on the observed degree of shadow cure. It was concluded that shadow cure may be enhanced if one or more fluorescent compounds emit fluorescent light at wavelengths absorbed by the dye in a two- or three-component photoinitiator system. The addition of DPI to the two-component systems containing MDEA and FL or EYss led to a significant enhancement in the observed shadow cure. This result was attributed to the fact that DPI will increase both the number of active centers and the mobility of the active centers as a result of the electron transfer reactions in which it participates.
\end{abstract}

\section{Introduction}

Photopolymerization has received considerable attention due to its environmental and processing advantages $[1,2]$. Compared to traditional thermal polymerization processes, light-induced polymerization offers reduced volatile organic compound emissions, lower energy requirements, and shorter cure times. Furthermore, light sources are generally much more compact than ovens and autoclaves used for thermal cure. For these reasons photopolymerization can be used not only for the replacement of conventional thermal processes but also for new systems and applications. In addition, the recent development of initiators for visible-light-induced photopolymerization enables processes based upon inexpensive light sources and lower energy photons which are not damaging to biological tissues and cells. Due to these advantages, visible-light-induced photopolymerization has been employed for various applications including coatings, adhesives, and printing. Despite these advantages, the inability to cure shaded regions which are inaccessible to the initiating light is a limitation for many applications. If this disadvantage is overcome a number of new applications of photopolymerization could emerge, such as three-dimensional adhesives [3], optical impact films [4], solar-light-induced coatings, and pigmented systems.

In photopolymerization systems, "shadow cure" may be defined as polymerization which extends into regions which are not illuminated by the incident initiating light source. Dual-cure systems containing both photoinitiators and thermal initiators for thick shadow cure are well known $[5,6]$; however, shadow cure in systems containing only photoinitiators has only recently been reported by Ficek et al. [7]. These authors demonstrated cationic photopolymerizations of thick systems in which polymerization could occur in shadow regions due to the mobility of the long-lived cationic active centers. Although this method could be attractive for some systems and applications, it has a number of limitations. It may only be applied to cationically polymerizable monomers, exhibits relatively slow cure rates compared to free radical systems, and the photo-generated protons may be corrosive to electronic devices. Therefore, in this contribution, shadow cure in free radical photopolymerizations 
<smiles>O=C(O)c1ccccc1-c1c2ccc(=O)cc-2oc2cc(O)ccc12</smiles>

(a)<smiles>O=C(O)c1ccccc1-c1c2cc(Br)c(=O)c(Br)c-2oc2c(Br)c(O)c(Br)cc12</smiles>

(b)<smiles>O=C(c1ccccc1)c1ccccc1</smiles>

(c)<smiles>CN(CCO)CCO</smiles>

(d)<smiles>Cl[I-](c1ccccc1)c1ccccc1</smiles>

(e)

FIGURE 1: Chemical structures of (a) fluorescein (FL), (b) eosin Y spirit soluble (EYss), (c) benzophenone (BP), (d) N-methyldiethanolamine (MDEA), (e) diphenyl iodonium chloride (DPI).

initiated using two-component and three-component photoinitiators is investigated.

Multicomponent photoinitiator systems are commonly employed for visible-light-induced photopolymerization. The energy of a visible photon is generally lower than the bond dissociation energy of most organic molecules; therefore visible-light-induced photoinitiator systems are primarily two-component photoinitiator systems in which the active centers are produced via an electron transfer followed by a proton transfer from the electron donor (typically an amine) to the excited photosensitizer [1]. Many dyes and other compounds that absorb in the visible range have been used as the photosensitizer in this type of system, including camphorquinone, (thio)xanthone derivatives, (thio)xanthene derivatives, and ketocoumarin derivatives [8-13]. In order to enhance the electron transfer system by adding other reaction schemes including diphenyl iodonium chloride (DPI) into these two-component photoinitiator systems, three-component photoinitiator systems have been developed and investigated as well [14-22].

The objective of this study is to evaluate the use of fluorescent additives for polymerization in masked regions that are unilluminated by the incident initiating light. In this method, the absorption of light by the dye molecules leads to emission of fluorescent light at a longer wavelength in all directions. Therefore, careful selection of the combination of the incident wavelength, the fluorescent additive, and the photosensitizer can lead to effective illumination and polymerization in shaded regions that are inaccessible to the incident light source. In this study, two different fluorescent dyes are investigated: fluorescein (FL) and eosin Y spirit soluble (EYss). Each of these dyes may form free radical active centers in two-component initiator systems containing $\mathrm{N}$ methyldiethanolamine (MDEA) or three-component initiator systems containing MDEA and DPI. A systematic series of studies was performed to characterize the effects of fluorescence intensity, the incident light intensity, and the presence of a diphenyl iodonium salt on the observed degree of shadow cure.

\section{Experimental}

2.1. Materials. A monomer mixture of $50 \mathrm{wt} \%$ 2-hydroxyethyl acrylate (Sigma-Aldrich) and $50 \mathrm{wt} \%$ 1,6-hexanediol diacrylate (Sartomer) was used in these experiments. This monomer mixture was selected because it dissolves photoinitiators well and leads to a rigid, highly crosslinked polymer. The multicomponent photoinitiator system materials FL, EYss, benzophenone (BP), MDEA, and DPI were obtained from Sigma-Aldrich. The molecular structures are displayed in Figure 1. The dyes chosen for this study have potential for fluorescence-derived shadow cure since there is overlap between the absorbance and emission spectra. For example, the fluorescein absorbance and emission spectra overlap at wavelengths between $475 \mathrm{~nm}$ and $510 \mathrm{~nm}$, and the eosin Y absorbance and emission spectra overlap at wavelengths between $500 \mathrm{~nm}$ and $550 \mathrm{~nm}$. At dye concentrations higher than $10{ }^{5} \mathrm{M}$, the dyes exist primarily as dimers, and the dimer formation is evident from the UV-visible absorption spectrum. For example, in the spectrum of fluorescein, the unassociated dye exhibits its maximum absorbance at a wavelength of $480 \mathrm{~nm}$, and the formation of the associated dye (dimer) leads to appearance of a blue shift with a maximum absorbance at $451 \mathrm{~nm}$ [23]. Similarly, in the spectrum of eosin $\mathrm{Y}$, the unassociated dye exhibits its maximum absorbance at a wavelength of $533 \mathrm{~nm}$, and the formation of the associated dye (dimer) leads to appearance of a minor blueshifted contribution at $495 \mathrm{~nm}$ [23-25]. At the concentration used for the shadow cure experiments $\left(1.15 \times 10^{3} \mathrm{M}\right)$, the maximum molar absorption coefficients (Napierian molar absorptivity) are $1,144 \mathrm{~L} / \mathrm{mol}-\mathrm{cm}$ at $451 \mathrm{~nm}$ wavelength for $\mathrm{FL}$ and $28,918 \mathrm{~L} / \mathrm{mol}-\mathrm{cm}$ at $533 \mathrm{~nm}$ wavelength for EYss. 


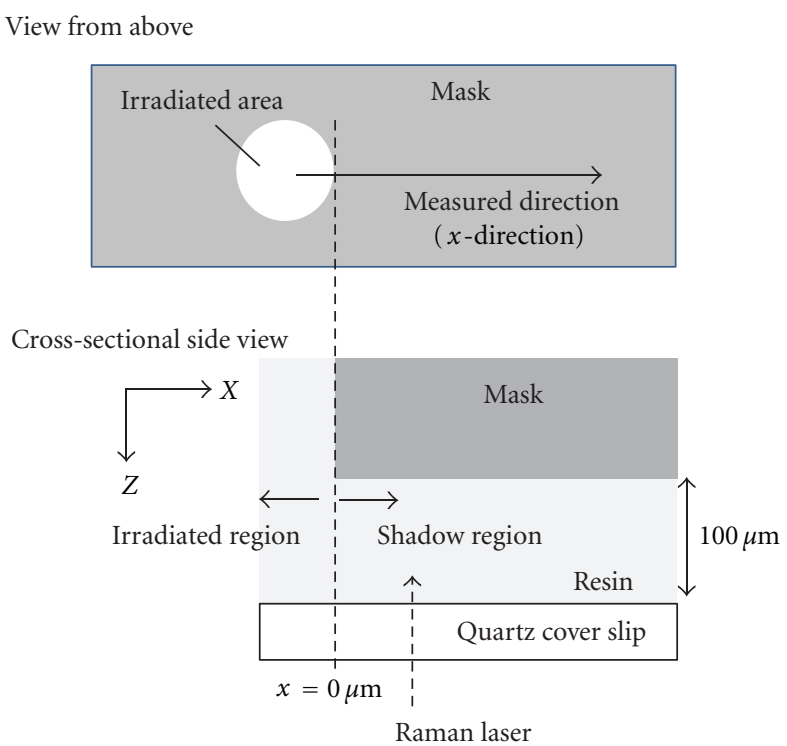

FIGURE 2: Schematic of the experimental geometry for the shadow cure studies. The mask was a $500 \mu \mathrm{m}$ thick steel plate with a $6 \mathrm{~mm}$ diameter hole through which the monomer mixture was illuminated from above. After illumination of the prescribed duration, Raman microscopy was used to collect line ( $x$-direction) and depth ( $z$-direction) conversion profiles through the quartz cover slip on the bottom of the sample.

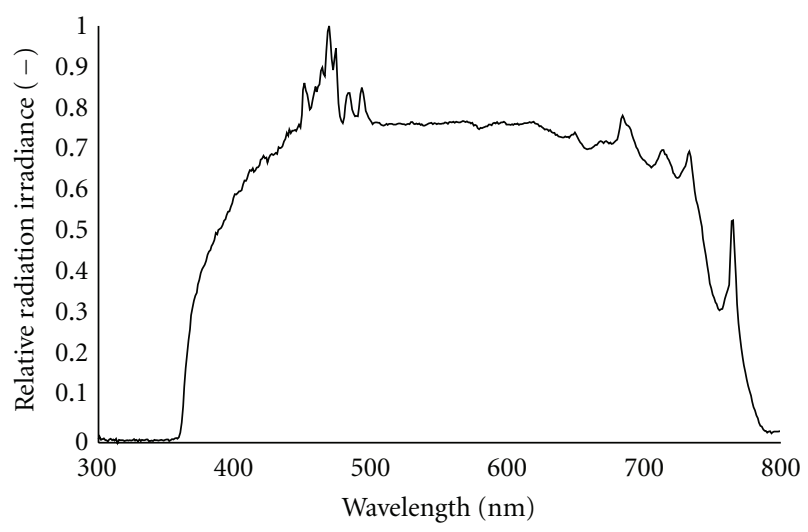

FIgURe 3: Emission spectrum of a $150 \mathrm{~W}$ xenon lamp.

Benzophenone has relatively low absorptivity above $300 \mathrm{~nm}$ wavelength and its molar absorption coefficient at $350 \mathrm{~nm}$ wavelength is $232 \mathrm{~L} / \mathrm{mol}-\mathrm{cm}$. Note that MDEA and DPI absorb only in the deep ultraviolet region of the spectrum and do not affect the absorption profile for wavelengths above $300 \mathrm{~nm}$.

2.2. Shadow Cure via Photopolymerization. Shadow cure experiments were performed in the simple reaction geometry shown in Figure 2. The mask was a $500 \mu \mathrm{m}$ thick steel plate with a $6 \mathrm{~mm}$ diameter hole through which the HEA-HDDA monomer mixture was illuminated using a $150 \mathrm{~W}$ xenon lamp (MAX-150, Asahi Spectra). The emission spectrum of this lamp is illustrated in Figure 3. Various multicomponent photoinitiator systems including dye/amine systems and dye/amine/DPI systems were used to photopolymerize $100 \mu \mathrm{m}$ thick HEA-HDDA monomer films. The compositions of the photoinitiator systems are summarized in Table 1. The monomer mixture containing the photoinitiator was sandwiched between a steel plate on the top surface and a quartz cover slip on the bottom surface. A $100 \mu \mathrm{m}$ thick spacer (PET film) was used to ensure a uniform film thickness for all experiments. The hole in the steel plate was capped by a $37 \mu \mathrm{m}$ thick PET film to avoid the influence of oxygen inhibition. Finally, the entire system was placed on a black stage to minimize reflection from the bottom and was illuminated from the top down for fifteen minutes. The xenon lamp emits in a broad region between $350 \mathrm{~nm}$ and $760 \mathrm{~nm}$, and the total incident light intensity was measured by a USB 4000 UV-VIS fiber optic spectrometer (Ocean Optics). The total intensity was varied by controlling the distance between the xenon lamp and the surface of the mask. This distance was $10 \mathrm{~cm}$ for an incident light intensity of $89 \mathrm{~mW} / \mathrm{cm}^{2}$ and as $2.5 \mathrm{~cm}$ for an incident light intensity of $1.0 \mathrm{~W} / \mathrm{cm}^{2}$.

2.3. Characterization of Shadow Cure. The monomer conversions in the shadow regions behind the steel plate were investigated through a quartz cover slip. As shown in Figure 2, the line ( $x$-direction) and depth ( $z$-direction) conversion profiles of the cured samples were measured using Raman microscopy. Raman spectra of the samples were collected using a Leica DLILP optical microscope with confocal optics attached to the modular Raman spectrograph (HoloLab 5000R, Kaiser Optical Systems, Inc) equipped with a $785 \mathrm{~nm}$ laser [26-28]. Note that the $785 \mathrm{~nm}$ laser does not cause photopolymerization for all samples. A combination of $785 \mathrm{~nm}$ single-mode excitation fiber, $100 \mu \mathrm{m}$ collection fiber, and $100 \mathrm{x}$ objective with numerical aperture equal to 0.9 was employed in the measurement. The laser intensity through the $100 \mathrm{x}$ lens was $7-12 \mathrm{~mW} / \mathrm{cm}^{2}$, measured by a laser power meter. For each measurement, the exposure time was set to 20-60 seconds with one accumulation in order to optimize the signal-noise ratio. The reactive $\mathrm{C}=\mathrm{C}$ peak at $1640 \mathrm{~cm}^{-1}$ and an unchanging reference peak at $1720 \mathrm{~cm}^{-1}$ (attributed to the $\mathrm{C}=\mathrm{O}$ carbonyl stretch) were monitored to determine the acrylate conversion. The conversion was calculated using the ratio of reactive $\left(A_{\mathrm{rxn}}\right)$ peak and internal reference $\left(A_{\text {ref }}\right)$ peak areas:

$$
\% \text { Conversion }=\left[1-\frac{A_{\text {rxn }}(p) / A_{\text {ref }}(p)}{A_{0}(u) / A_{\text {ref }}(u)}\right] \times 100
$$

where " $u$ " refers to unpolymerized and " $p$ " to polymerized resin.

The conversion profiles in the $x$-direction, perpendicular to the edge of the illuminated region, were measured by performing a line scan. The conversion profile beginning in the illuminated region and extending into the shadow region was characterized by taking measurements at $40 \mu \mathrm{m}$ increments for distances up to $5,000 \mu \mathrm{m}$ from the illuminated edge. The precision of the conversion measurements was generally $\pm 3 \%$. Confocal Raman spectroscopy was used to verify that the conversion was uniform across the thickness of 


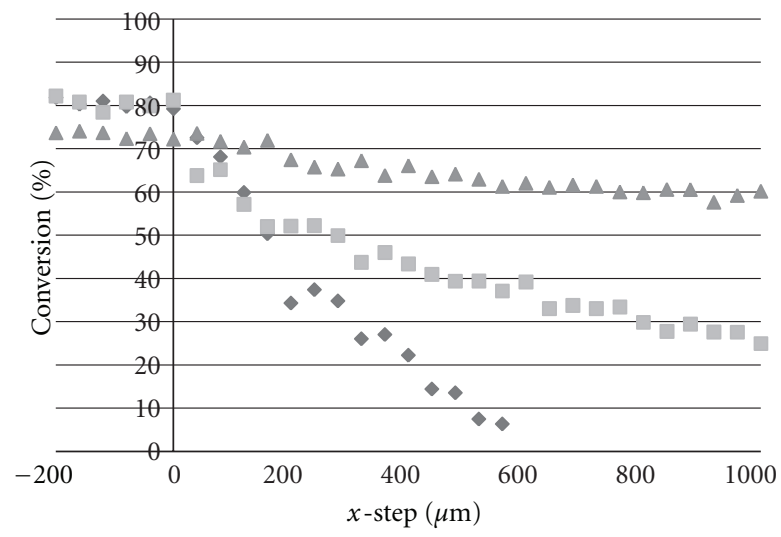

$$
\begin{aligned}
& \text { BP/MDEA } \\
& \text { FL/MDEA } \\
& \text { EYss/MDEA }
\end{aligned}
$$

FIGURE 4: Shadow cure conversion profiles ( $x$-direction) resulting from three different dye/amine photoinitiator systems. Negative values of $x$ correspond to the illuminated region, while positive values of $x$ correspond to the masked region. Light source: a $150 \mathrm{~W}$ xenon lamp, light

\begin{tabular}{|c|c|c|c|c|c|}
\hline \multirow{2}{*}{ Systems } & \multicolumn{3}{|c|}{ Dyes } & \multirow{2}{*}{$\begin{array}{c}\text { Amine } \\
\text { MDEA (M) }\end{array}$} & \multirow{2}{*}{$\begin{array}{c}\text { Iodonium salt } \\
\text { DPI (M) }\end{array}$} \\
\hline & $\mathrm{BP}(\mathrm{M})$ & $\mathrm{FL}(\mathrm{M})$ & EYss (M) & & \\
\hline BP/MDEA & 0.00115 & - & - & 0.32 & - \\
\hline FL/MDEA & - & 0.00115 & - & 0.32 & - \\
\hline EYss/MDEA & - & - & 0.00115 & 0.32 & - \\
\hline FL/MDEA/DPI & - & 0.00115 & - & 0.32 & 0.0023 \\
\hline EYss/MDEA/DPI & - & - & 0.00115 & 0.32 & 0.0023 \\
\hline
\end{tabular}
intensity: $89 \mathrm{~mW} / \mathrm{cm}^{2}$, irradiation time: 15 minutes.

TABLE 1: Molar concentration of components in the photoinitiator systems under investigation.

the sample (the $z$-direction). This result is not surprising due to the relatively small thickness of this illuminated region. Therefore, only the line profiles in the $x$-direction, perpendicular to the edge of the illuminated region, will be considered in the remainder of this paper.

\section{Results and Discussion}

3.1. Shadow Cure with Dye/Amine Photoinitiator Systems. Figure 4 contains plots of the conversion profiles in the $x$-direction, perpendicular to the edge of the illuminated region for three different photoinitiator systems: BP/MDEA, FL/MDEA, and EYss/MDEA. In this figure, the origin occurs at the illuminated edge; therefore negative values of the abscissa correspond to the illuminated region. The conversion in the illuminated region was relatively constant, therefore only the first $200 \mu \mathrm{m}$ are shown in the figure. For all three initiator systems, the conversion in the illuminated region is above $70 \%$. The figure illustrates that, as the scanned position moves away from the boundary $(x=0)$, the conversion decreases at a rate that depends upon the initiator system. For example, the conversion of the BP/MDEA system decreases to a value of zero in approximately $500 \mu \mathrm{m}$, while at $500 \mu \mathrm{m}$ the FL/MDEA and EYss/MDEA systems exhibit conversions of $40 \%$ and $64 \%$, respectively. The data in Figure 4 illustrate that the photoinitiator system has a marked effect on the observed degree of shadow cure and that the systems exhibit the following trend: BP/MDEA $<$ FL/MDEA $<$ EYss/MDEA. Note that, for the EYss/MDEA, even at the distance of $1,000 \mu \mathrm{m}$ from the illuminated edge, the conversion is approximately $60 \%$.

The shadow cure in the masked region illustrated in Figure 4 could arise from a number of effects, including scattering of the incident light, reflection from the quartz cover slip at the bottom surface, or diffusion of active centers into the shadow region. The shadow cure observed for the BP/ MDEA system is likely to arise only from these effects since BP emits negligible fluorescence. For systems containing fluorescein or eosin Y spirit soluble, fluorescence light from the dyes may also enhance the shadow cure since dye molecules that absorb light in the illuminated region will fluoresce in all directions. Absorption of this fluorescent light by dye molecules in the shadow region may lead to active center generation. The dyes chosen for this study have potential for fluorescence-derived shadow cure since there is overlap between the absorbance and emission spectra. As mentioned in Section 2.1, the fluorescein absorbance and emission spectra overlap at wavelengths between $475 \mathrm{~nm}$ and $510 \mathrm{~nm}$, and the eosin $\mathrm{Y}$ absorbance and emission spectra overlap at wavelengths between $500 \mathrm{~nm}$ and $550 \mathrm{~nm}$. 


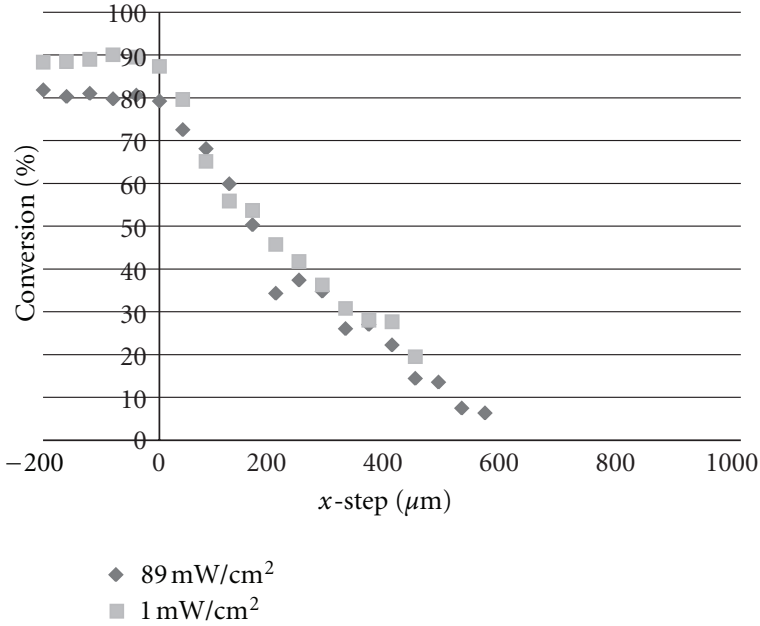

(a)

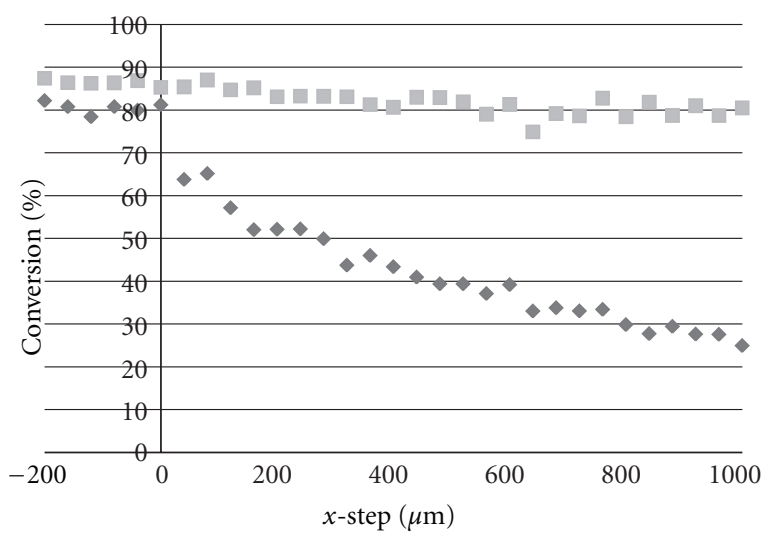

- $89 \mathrm{~mW} / \mathrm{cm}^{2}$

$1 \mathrm{~mW} / \mathrm{cm}^{2}$

(b)

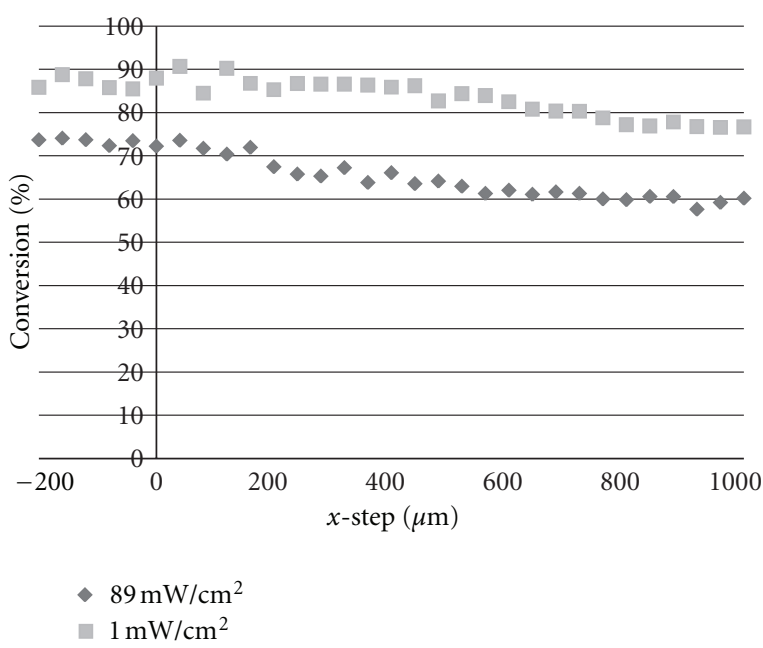

(c)

FIGURE 5: The effect of the incident light intensity on the conversion profiles ( $x$-direction) observed for three different dye/amine systems: (a) BP/MDEA, (b) FL/MDEA, (c) EYss/MDEA. Light source: a $150 \mathrm{~W}$ xenon lamp, irradiation time: 15 minutes.

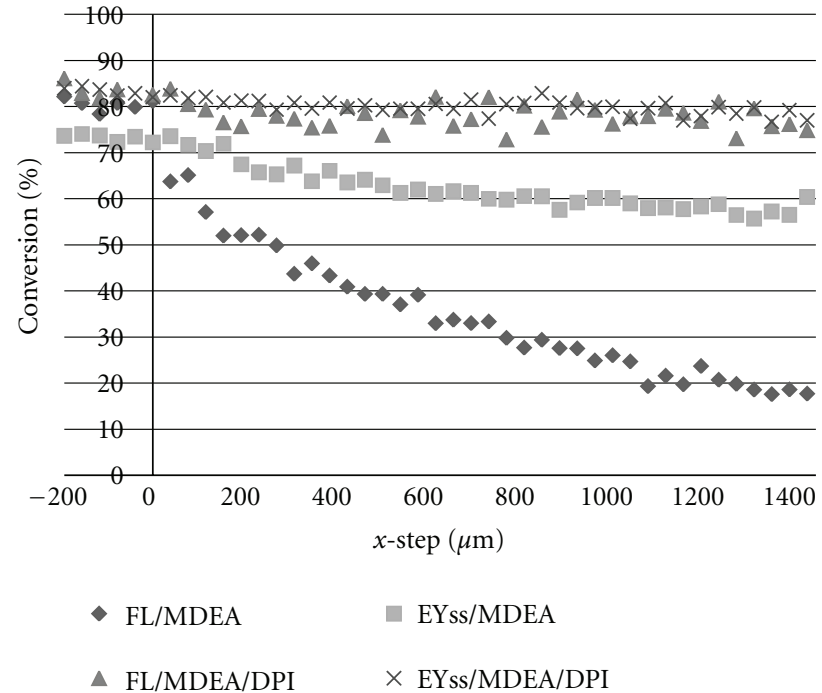

FIGURE 6: Effect of the addition of DPI on the shadow cure conversion profiles ( $x$-direction) observed for FL and EYss dye/amine photoinitiator systems. Light source: a $150 \mathrm{~W}$ xenon lamp, light intensity: $89 \mathrm{~mW} / \mathrm{cm}^{2}$, irradiation time: 15 minutes.

The shadow cure trends illustrated in Figure 4 are consistent with the hypothesis that fluorescent light from the dyes leads to active center generation in the shadow region. Benzophenone exhibits negligible fluorescence; therefore the profile obtained for the BP/MDEA system provides a "control" experiment to which the fluorescent systems can be compared. It is interesting that other fluorescence-free photoinitiators such as bis(2,4,6-trimethylbenzoyl)-phenylphosphine oxide and 1-hydroxy-cyclohexyl-phenyl-ketone yielded similar conversion profiles. For the FL/MDEA and EYss/MDEA systems, the total intensity of fluorescent light was calculated from first principles $[29,30]$ based upon the concentration of the dye, sample thickness, the intensity and spectrum of the incident light, the absorbance spectra of the respective dyes, and the fluorescence quantum yield. Note that the dyes exist primarily as associated dimers at the concentration used for the shadow cure experiments $(1.15 \times$ $10^{3} \mathrm{M}$ ); therefore the molar absorptivity coefficients of the dimers must be used [23-25]. This calculation revealed that the fluorescent light generated by eosin $\mathrm{Y}$ was 7.8 times more intense than the fluorescent light generated by fluorescein. Despite the high fluorescence quantum yield of fluorescein (0.83 [29]), the fluorescence emission into the shadow region for the FL/MDEA system is low compared to that of EYss/ MDEA due to the low molar absorption coefficient of fluorescein.

Figure 5 illustrates the effect of the incident light intensity on the conversion profiles and the observed extent of shadow cure for three different photoinitiator systems: BP/MDEA, FL/MDEA, and EYss/MDEA. The figure illustrates that the extent of shadow cure for the system which does not contain a fluorescent dye (BP/MDEA, Figure 5(a)) does not show any appreciable difference when the light intensity is increased. In contrast, the extent of shadow cure is significantly enhanced 


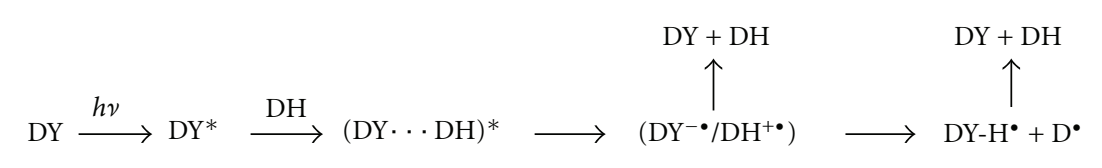

(a) Dye-amine reaction
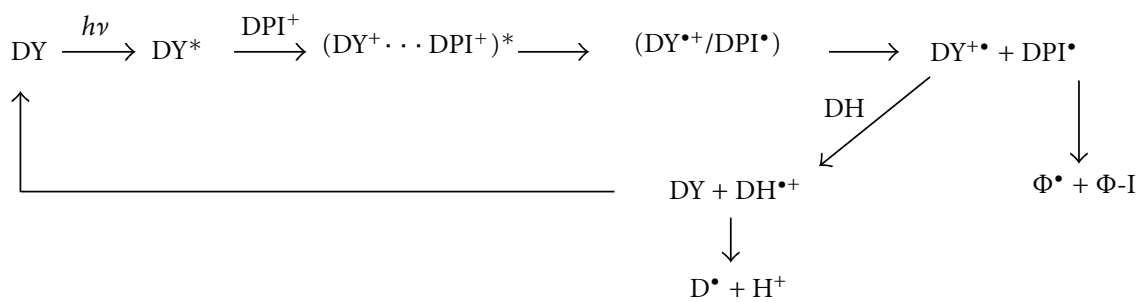

DY: dye(FL or EYss)

DH: electron donor (MDEA)

$\mathrm{DPI}^{+}$: iodonium salt
$\Phi^{\bullet}:$ phenyl radical

$\Phi-I$ : phenyl iodide

(b) Dye-DPI-amine reaction

FIGURE 7: Schematic representation of the reaction mechanism for three-component photoinitiator systems containing FL or EYss, MDEA, and DPI. (a) photoreduction of the FL or EYss dye by MDEA and (b) photooxidation of the FL or EYss dye by DPI.

for the systems containing the fluorescent dyes (FL/MDEA and EYss/MDEA in Figures 5(b) and 5(c), resp.). In both cases, for the high light intensity $\left(1.0 \mathrm{~W} / \mathrm{cm}^{2}\right)$ the cure in the shadow region is nearly equivalent to that in the illuminated region. It is worth noting that oxygen inhibition is small in these systems because they are not exposed to the atmosphere, and the initially dissolved oxygen is consumed by MDEA active centers through a well-known cyclic reaction [31]. These results suggest that the increased incident light intensity leads to increased fluorescence intensity, thereby resulting in increased shadow cure for the systems containing the fluorescent dye.

3.2. Shadow Cure with Dye/Amine/DPI Photoinitiator Systems. A series of experiments was performed to investigate the effect of the addition of diphenyl iodonium chloride (DPI) on the shadow cure observed for systems containing MDEA and FL or EYss. Figure 6 contains the conversion profiles obtained for the two-component initiator systems (FL/ MDEA and EYss/MDEA) and the corresponding threecomponent photoinitiator systems (EYss/MDEA/DPI and FL/MDEA/DPI). The figure illustrates that the addition of DPI leads to a significant enhancement in the shadow cure observed for both dyes. In both cases, the shadow cure conversion in the shadow region exhibits a relatively high and uniform value for a distance of $1500 \mu \mathrm{m}$ from the illuminated edge. For the three-component initiator systems, the conversion a few millimeters into the shadow region is nearly the same as that of the illuminated region.

To explain the effect of DPI on the observed degree of shadow cure, it is useful to consider the reaction mechanism which has been reported in the literature as shown in Figure $7[17,20]$. In this photoinitiator system, the dye in its excited state may react with either MDEA or DPI in the parallel reactions shown in Figure 7. As shown in Figure 7(a), free radical active centers $(\mathrm{D} \bullet)$ are produced by the electron transfer reaction from the MDEA (DH) to the excited state of the dye. As shown in Figure 7(b), DPI oxidizes an excited dye molecule in a reaction that produces two active centers (an MDEA radical and a phenyl radical $(\Phi \bullet)$ ). Also, the DPI may oxidize nearly any radical species, such as an immobile radical that is trapped in the crosslinked polymer network to produce a mobile active phenyl radical. Finally, as shown in Figure $7(\mathrm{~b})$, the oxidized dye $\left(\mathrm{DY}^{+} \bullet\right.$ ) may be reduced by MDEA to regenerate the dyes-this would enhance the fluorescence effect and produce mobile initiating radicals. In addition, the DPI can enhance the number of effective active centers (free radicals capable of propagating) from the amine/dye reactions by irreversibly oxidizing the $\mathrm{DY}^{-} \bullet$ radical and thereby preventing the back-electron transfer reaction shown in Figure 7(a). The reduced DPI will, in turn, dissociate to produce an active phenyl radical. This can be significant since the $\mathrm{DY}^{-} \bullet$ radical is generally not active for propagation. Therefore, the large impact of DPI on the observed extent of shadow cure likely arises from the effect of DPI increasing both the number of active centers and the mobility of the active centers.

\section{Conclusions}

The experimental results reported in this contribution demonstrate that shadow cure in free radical photopolymerizations of masked systems can be enhanced with the use of fluorescent additives in two- or three-component initiator systems. In this method, the absorption of light by the fluorescent additive leads to emission of fluorescent light at 
a longer wavelength in all directions, including into the shadow region. Enhanced shadow cure was observed if a fluorescent compound emitted fluorescent light at wavelengths absorbed by the dye in a two-component photoinitiator system as illustrated by the following shadow cure trend: BP/ MDEA < FL/MDEA < EYss/MDEA. The addition of DPI to the two-component systems containing MDEA and FL or EYss led to a significant enhancement in the observed shadow cure. This result was attributed to the fact that DPI will increase both the number of active centers and the mobility of the active centers as a result of the electron transfer reactions in which it participates. For example, DPI may oxidize an excited dye molecule in a reaction that produces two active centers; the oxidized dye may be reduced by MDEA to regenerate the dye (thereby enhancing the fluorescence), and DPI may oxidize immobile radicals that are trapped in the crosslinked polymer network to produce mobile active phenyl radicals.

\section{References}

[1] J. P. Fouassier, Photoinitiation, Photopolymerization, and Photocuring, Hanser, Munich, Germany, 1995.

[2] Y. Takimoto, Photopolymer Surface Fabrication Material, Bunshin Corporation, 2001.

[3] N. Sato, "Pre-irradiation system: applications," Three Bond Technical News, vol. 42, pp. 1-7, 1994.

[4] Y. Shinya, K. Kamiya, and Y. Kamata, "Image display device and its manufacturing method," US Patent, US2009/ 0186552A1, 12//309,106, 2009.

[5] S. Gregory, "Ultraviolet curable resin compositions having enhanced shadow cure properties," US Patent, 6245827, 2001.

[6] S. Hayashi, Y. Tasaka, N. Hayashi, and Y. Akita, "Development of smart polymer materials and its various applications," Mitsubishi Juko Giho, vol. 41, pp. 62-64, 2004.

[7] B. A. Ficek, A. M. Thiesen, and A. B. Scranton, "Cationic photopolymerizations of thick polymer systems: active center lifetime and mobility," European Polymer Journal, vol. 44, no. 1, pp. 98-105, 2008.

[8] J. D. Oxman, F. A. Ubel III, and E. G. Larson, "Coated abrasive binder containing ternary photoinitiator system," U.S. Patent, 4735632, 34066, 1988.

[9] M. Kawabata, K. Kimoto, and Y. Takimoto, "Photopolymerizable composition containing a certain xanthene or thioxanthene," US Patent, 4766055, 1988.

[10] J. P. Fouassier and E. Chesneau, "Polymerization induced by irradiation wih a visble laser, 4 . The system eosin UV-photoinitiator amine," Macromolecular Chemistry, vol. 192, pp. 245260, 1991.

[11] J. Fouassier and S. K. Wu, "Visible laser lights in photoinduced polymerization. VI. Thioxanthones and ketocoumarins as photoiniators," Journal of Applied Polymer Science, vol. 44, no. 10, pp. 1779-1786, 1992.

[12] R. Davidson, "The chemistry of photoinitiators—-some recent developments," Journal of Photochemistry and Photobiology A, vol. 73, no. 2, pp. 81-96, 1993.

[13] S. Jockusch, H. J. Timpe, W. Schnabel, and N. Turro, "Photoreduction of organic dyes in ketone amine systems," Journal of Photochemistry and Photobiology A, vol. 96, no. 1-3, pp. 129136, 1996.

[14] J. P. Fouassier, D. Ruhlmann, B. Graff, Y. Takimoto, M. Kawabata, and M. Harada, "New three-component system in visible laser light photo-induced polymerization," Journal of Imaging Science, vol. 37, no. 2, pp. 208-210, 1993.

[15] A. Erddalane, J. P. Fouassier, F. Morlet-Savary, and Y. Takimoto, "Efficiency and excited state processes in a three-component system, based on thioxanthene derived dye/amine/additive, usable in photopolymer plates," Journal of Polymer Science A, vol. 34, no. 4, pp. 633-642, 1996.

[16] K. S. Padon and A. B. Scranton, "Mechanistic investigation of a three-component radical photoinitiator system comprising methylene blue, $N$-methyldiethanolamine, and diphenyliodonium chloride," Journal of Polymer Science A, vol. 38, no. 11, pp. 2057-2066, 2000.

[17] D. Chloride, K. S. Padon, and A. B. Scranton, "A mechanistic investigation of the three-component radical photoinitiator system eosin y spirit soluble, n-methyldiethanolamine," Journal of Polymer Science A, vol. 39, no. 5, pp. 715-723, 2001.

[18] J. P. Fouassier, X. Allonas, and D. Burget, "Photopolymerization reactions under visible lights: principle, mechanisms and examples of applications," Progress in Organic Coatings, vol. 47, no. 1, pp. 16-36, 2003.

[19] D. Kim and A. B. Scranton, "The role of diphenyl iodonium salt (DPI) in three-component photoinitiator systems containing methylene blue (MB) and an electron donor," Journal of Polymer Science A, vol. 42, no. 23, pp. 5863-5871, 2004.

[20] D. Kim, A. B. Scranton, and J. W. Stansbury, "Analysis of association constant for ground-state dye-electron acceptor complex of photoinitiator systems and the association constant effect on the kinetics of visible-light-induced polymerizations," Journal of Polymer Science A, vol. 47, no. 5, pp. 1429-1439, 2009.

[21] D. Kim and J. W. Stansbury, "A photo-oxidizable kinetic pathway of three-component photoinitiator systems containing porphrin dye (Zn-tpp), an electron donor and diphenyl lodonium salt," Journal of Polymer Science A, vol. 47, no. 12, pp. 3131-3141, 2009.

[22] A. Ibrahim, C. Ley, O. I. Tarzi, J. P. Fouassier, and X. Allonas, "Visible light photoinitiating systems: toward a good control of the photopolymerization efficiency," Journal of Photopolymer Science and Technology, vol. 23, no. 1, pp. 101-108, 2010.

[23] S. Speiser and F. L. Chisena, "Optical bistability in fluorescein dyes," Applied Physics B, vol. 45, no. 3, pp. 137-144, 1988.

[24] R. W. Chambers, T. Kajiwara, and D. R. Kearns, "Effect of dimer formation of the electronic absorption and emission spectra of ionic dyes. Rhodamines and other common dyes," Journal of Physical Chemistry, vol. 78, no. 4, pp. 380-387, 1974.

[25] D. Fornasiero and T. Kurucsev, "Vibronic exciton bands. Absorption spectra of Eosin Y dimers," Journal of the Chemical Society, vol. 82, no. 1, pp. 15-19, 1986.

[26] Y. Cai and J. L. P. Jessop, "Decreased oxygen inhibition in photopolymerized acrylate/epoxide hybrid polymer coatings as demonstrated by Raman spectroscopy," Polymer, vol. 47, no. 19, pp. 6560-6566, 2006.

[27] Y. Zou, S. R. Armstrong, and J. L. P. Jessop, "Apparent conversion of adhesive resin in the hybrid layer, part 1: identification of an internal reference for Raman spectroscopy and the effects of water storage," Journal of Biomedical Materials Research A, vol. 86, no. 4, pp. 883-891, 2008.

[28] Y. Zou, J. L. P. Jessop, and S. R. Armstrong, "Apparent conversion of adhesive resin in the hybrid layer, part II: in situ studies of the resin-dentin bond," Journal of Biomedical Materials Research $A$, vol. 89, no. 2, pp. 355-362, 2009.

[29] L. S. Forster and D. Dudley, "The luminescence of fluorescein dyes," Journal of Physical Chemistry, vol. 66, no. 5, pp. 838-840, 1962. 
[30] N. S. Kenning, B. A. Ficek, C. C. Hoppe, and A. B. Scranton, "Spatial and temporal evolution of the photoinitiation rate for thick polymer systems illuminated by polychromatic light: selection of efficient photoinitiators for LED or mercury lamps," Polymer International, vol. 57, no. 10, pp. 1134-1140, 2008.

[31] K. S. Padon and A. B. Scranton, "The effect of oxygen on the three-component radical photoinitiator system: methylene blue, $N$-methyldiethanolamine, and diphenyliodonium chloride," Journal of Polymer Science A, vol. 38, no. 18, pp. 3336$3346,2000$. 


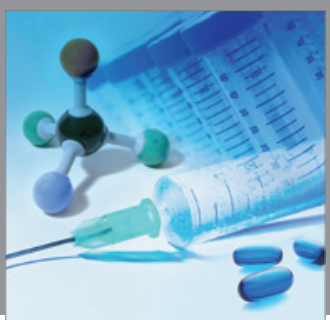

International Journal of

Medicinal Chemistry

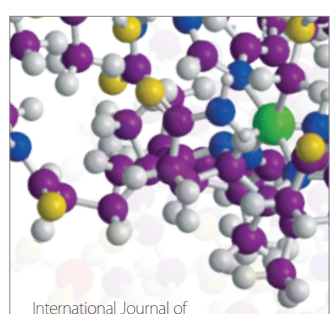

Carbohydrate Chemistry

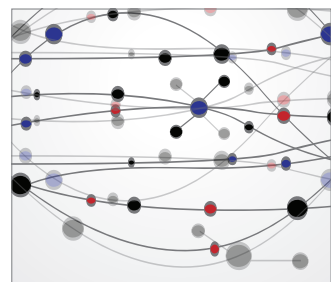

The Scientific World Journal
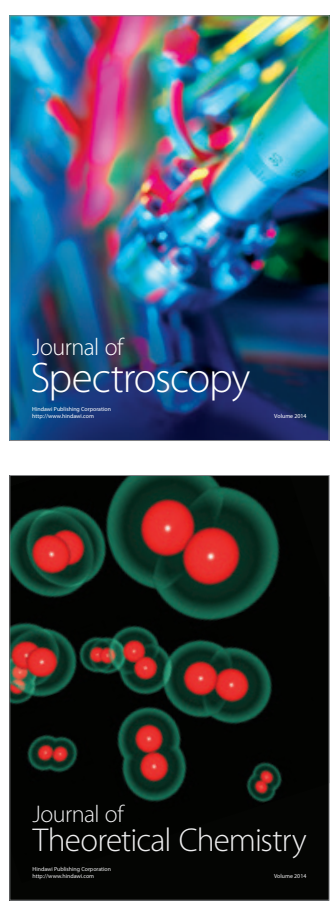
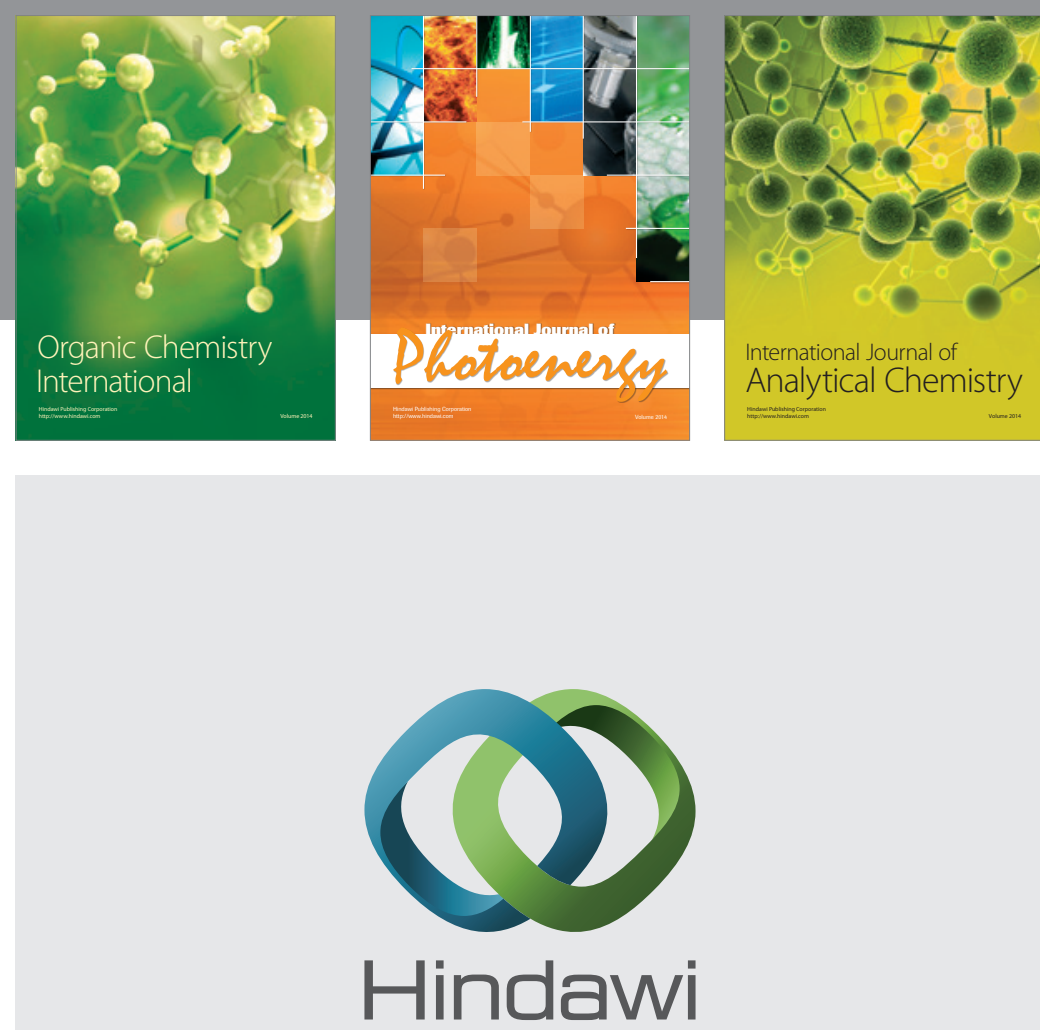

Submit your manuscripts at

http://www.hindawi.com
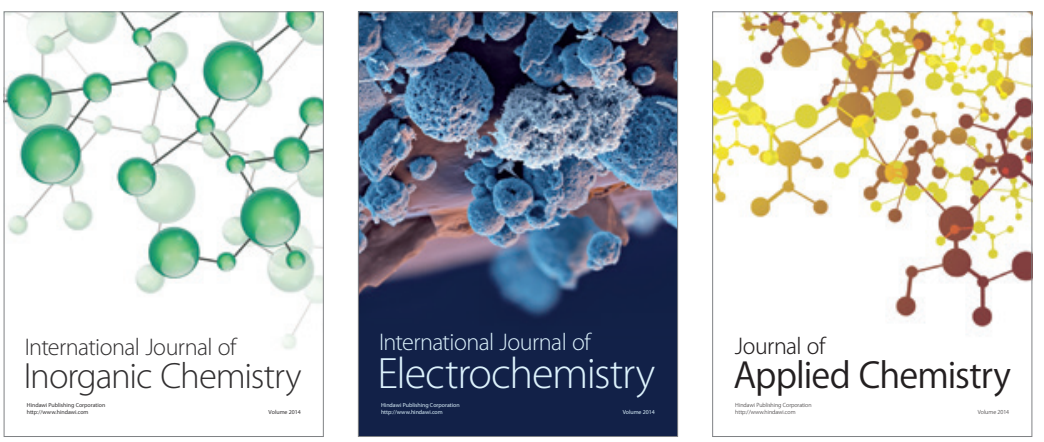

Journal of

Applied Chemistry
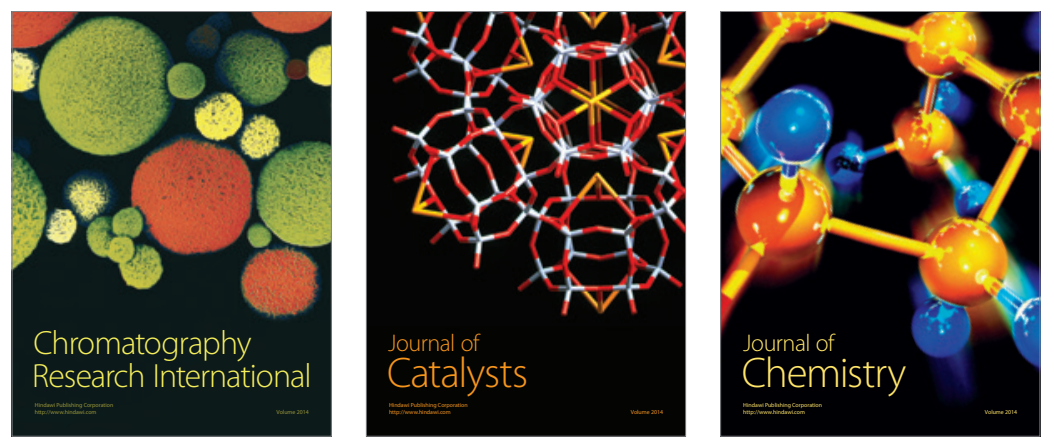
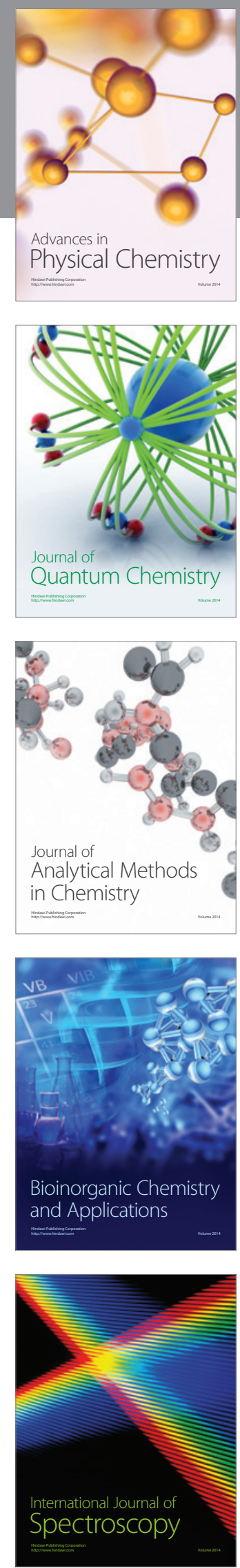